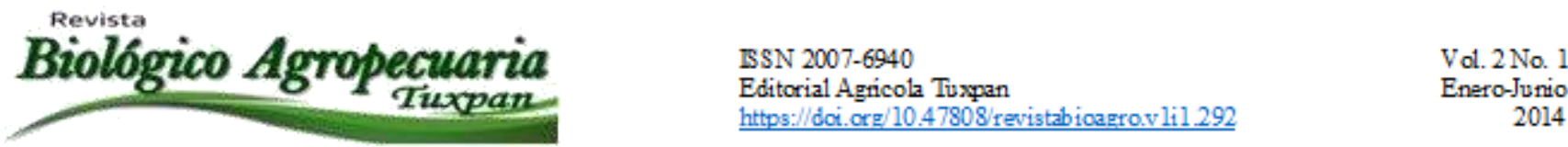

\title{
Estudio físico-químico en salchichas adicionadas con almidón de plátano macho (Musa paradisiaca)
}

Physico-chemical study sausages with added plantain starch

\author{
Sánchez Rodríguez J. F. ${ }^{1}$, S. Soto-Simental ${ }^{2}$ y N. Güemes-Vera ${ }^{\circledR}$
}

${ }^{1}$ Facultad de Agronomía-Universidad Autónoma de Sinaloa, km 17.5 carretera a Costa Rica Apdo. postal Núm. 723 Culiacán, Sinaloa. ${ }^{2}$ Instituto de Ciencias Agropecuarias-Área Académica de Ingeniería Agroindustrial e Ingeniería en Alimentos Universidad Autónoma del Estado de Hidalgo. Av. Universidad Km 1. Ex Hacienda de Aquetzalpa Tulancingo Hidalgo C.P. 43600.

${ }^{凶}$ Autor para correspondencia: njgv2002@yahoo.com.mx

Recibido: $26 / 01 / 2014$

Aceptado: 17/07/2014

\section{RESUMEN}

El objetivo de este trabajo fue adicionar harina de plátano macho, la cual contiene, almidones, minerales (potasio) y fibras, con la finalidad de que este contenido sustituya al que se adiciona de forma convencional en las formulaciones de salchichas, con el propósito de obtener salchichas con buenas propiedades nutricionales, físicas y sensoriales. Se encontró que las salchichas adicionadas con harina de plátano en proporciones de $0,20,30$ y 40\%, sustituyendo a la harina de maíz, presentó una dureza mayor con diferencia significativa $(\mathrm{P}<0.0 .1)$ en todos los porcentajes de adición con respecto al testigo. La cohesividad y elasticidad no tuvieron diferencia alguna ( $\mathrm{P}>0.05)$, en cambio la adhesividad incrementó conforme aumentaba la adición de harina de plátano. Al probar la prueba de textura con la navaja Watner-Blatzer, se encontró una disminución en el esfuerzo al corte, conforme se aumento la concentración de harina de plátano. La Aw no tuvo ningún cambio por la adición de esta, no fue así en la capacidad de retención de agua la cual fue aumentando de una forma ligera hasta el $30 \%$ de incorporación, para posteriormente bajar en el $40 \%$. Con respecto al color este fue disminuyendo. Se realizó la prueba de sensorial a 35 jueces no entrenados, de los cuales el $70 \%$ les gusto el producto y al $30 \%$ restante les gusto mucho.

\section{ABSTRACT}

The aim of this work was to add banana flour, this flour content, starches, minerals (potassium) and fiber, the purpose of this content is added to replace the conventional way in the formulations of sausages, in order to obtain sausages with good nutritional properties, physical and sensory. It was 


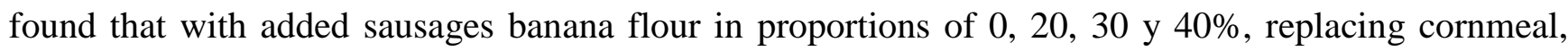
presented higher hardness with significant difference $(\mathrm{P}<0.0 .1)$ in addition all percentages relative to the control. The cohesiveness and springiness had no difference $(\mathrm{P}>0.05)$, however the increased adhesiveness with increasing the addition of banana flour. When testing with test texture WatnerBlatzer knife, found a decrease in the shear, as they increase the concentration of banana flour. The Aw had not change by adding this, it was not in the water holding capacity which was in a slight increase to $30 \%$ incorporation, to subsequently fall in the $40 \%$. With respect to color this was declining. We performed sensory testing 35 untrained judges, of which $70 \%$ they like the product and the remaining $30 \%$ like them a lot.

Keywords: sausage, banana, texture, water activity.

\section{INTRODUCCION}

Los plátanos (Musa paradisiaca) son considerados uno de los primeros frutos en ser cosechados por los agricultores primitivos y han sido presentados en diversas culturas y civilizaciones por cientos de años. Los plátanos son originarios del sureste de Asia, incluyendo el norte de la india, Cambodia, Sumatra, Java, Las Filipinas y Taiwán. En los años sesenta el cultivo de plátano fue introducido en las islas de santo domingo y cuba. A finales de los años noventa, las primeras plantaciones comerciales fueron establecidas en Jamaica y después en diversos países de América central y México (Bello, 2011).

La composición química del plátano depende del estado de maduración, sin embargo, las características agronómicas como el tipo de suelo y las condiciones climáticas alteran mayor o menormente los componentes de la fruta. En un estudio la menor humedad contenida, fue encontrada en la pulpa inmadura del plátano (69\%) y en la pulpa del plátano maduro fue de (74\%), los carbohidratos contenidos fueron más altos en el plátano inmaduro (28\%) y en el plátano maduro $(21 \%)$. Pero un patrón inverso fue obtenido por la fibra. La pulpa inmadura tuvo $2 \%$ y la pulpa madura tuvo $5 \%$, este patrón pudo haber sido obtenido por los altos niveles de pectina presentes en la pulpa en estado maduro (Bello, 2011).

Los almidones que más se utilizan para la elaboración de salchichas son aquellos que se obtienen del maíz, papa, y en otros casos se usan almidones modificados, de yuca o cassava dependiendo de costo y manejabilidad en el proceso. Por lo general estos tienen efectos en las salchichas como el de disminuir el $\mathrm{pH}$, contenido de agua y las pérdidas de peso durante la cocción, mostrando una mayor estabilidad al sistema. Lo que respecta a la textura de estos productos, se incrementan los valores de compresión la dureza, elasticidad y Cohesividad (Rivera R.I.N, 2012).

El objetivo de este trabajo fue elaborar salchichas de carne de cerdo y res, y adicionarlas con harina de plátano macho en diferentes proporciones, sin alterar las propiedades, físicas, química y sensoriales del producto.

\section{MATERIALES Y MÉTODOS}

\author{
Elaboración de las salchichas
}


Existo un reemplazo de almidón de plátano por carne, para observar el efecto que tienen estos almidones como ligantes.

\section{Análisis de perfil de textura en salchichas}

A las salchichas se les realizó un análisis de perfil de textura con un texturómetro TA-HDi, los accesorios usados los especiales para APT. Las condiciones de prueba para el APT: celda de carga $5 \mathrm{~kg}$, distancia 10.00 $\mathrm{mm} / \mathrm{s}$, velocidad de ensayo $1.00 \mathrm{~mm} / \mathrm{s}$.

\section{Fuerza máxima al corte}

La fuerza máxima reportada se determinó con la navaja de Warner Bratzler, para ello esta sonda se adaptó a la celda de un equipo analizador de textura TA-HDi (Textura Technologies, Stable Microsystems, Surrey, UK), con una celda de carga de $50 \mathrm{Kg}$., las condiciones de programación del equipo fueron una velocidad durante la prueba de $1 \mathrm{~mm} / \mathrm{s}$. La preparación de las muestras fue la siguiente: 1 $\mathrm{cm}$. de longitud, $1 \mathrm{~cm}$. grosor y $1 \mathrm{~cm}$. de ancho, la muestra se coloco horizontalmente entre la base de la celda, la cual bajo verticalmente hasta lograr la completa penetración de la muestra.

\section{Actividad de agua}

Se prepararon las soluciones saturadas de las siguientes sales: cloruro de $\mathrm{Li}$, acetato de $\mathrm{K}$, cloruro de $\mathrm{Mg}$, carbonato de $\mathrm{K}$ nitrato de $\mathrm{Mg}$, cloruro de $\mathrm{Na}$, cloruro de $\mathrm{K}$ y nitrito de sodio. Se cortaron tiras de papel filtro de $(0.5 \times 2.5 \mathrm{~cm})$, cuidadosamente se tomaron las tiras con ayuda de unas pinzas, se humedecieron con la solución correspondiente se colocaron dos tiras dentro de una caja petri de vidrio, se dejaron secar a temperatura ambiente, una vez secas se colocó la muestra en la base de la caja (evitando que el alimento o muestra tenga contacto con las tiras) se procedió a sellar la caja con cinta adhesiva para evitar que la humedad del ambiente afecte. Después de $24 \mathrm{~h}$ se observaron las muestras que presentaron humedad y en función de las sales se determinó cual es la Aw.

\section{Capacidad de retención de agua}

Se determinó utilizando el método de Jauregui y col. (1981), se pesaron piezas de papel Whatman \#1 y $5.5 \mathrm{~cm}$ de diámetro doblado en forma de cono y se colocaron en el mismo $1.5 \mathrm{~g}$ de muestra. Los conos de papel con muestra fueron colocados en tubos de ensayo para centrifugarse a $3500 \mathrm{~g}$ por $15 \mathrm{~min}$. Transcurrido este tiempo se retiraron las muestras del papel y se pesaron. La capacidad de retención de agua fue reportada como el peso perdido con relación al peso inicial de la muestra, antes de centrifugar, expresada en porcentaje.

\section{Determinación de color}

El color en las muestras se determinó a través de los parámetros $\mathrm{L}^{*}, \mathrm{a}^{*}$ y $\mathrm{b}^{*}$, utilizando un colorímetro Minolta modelo 508d, con iluminante $\mathrm{C}$ y un ángulo observador de $10^{\circ}$. Se realizaron 3 mediciones de color sobre la superficie de cada salchicha con almidón de plátano. Se registraron los valores de $\mathrm{L}^{*}, \mathrm{a}^{*} \mathrm{y}$ $\mathrm{b}^{*}$, con los cuales se calcularon la cromaticidad $\left(\mathrm{C}^{*}\right)$ y el ángulo de matiz $\left(\mathrm{h}^{\circ}\right)$.

\section{Evaluación sensorial del producto}

El producto final fue evaluado sensorialmente mediante un prueba afectiva descrita por (Alzaldúa-Morales, 1994), de nivel de agrado, usando una escala hedónica de 9 puntos, utilizando un panel no entrenado de 35 jueces; genero mixto entre $18-25$ años de edad. 
A cada juez se le proporcionara una muestra de $10 \mathrm{~g}$ de salchicha a temperatura ambiente.

\section{Diseño experimental y Análisis estadístico}

Para esta investigación se decidió hacer 4 tratamientos con diferentes cantidades de almidón de plátano, las cantidades que se sustituyeron fueron del 10, 20, 30 y $40 \%$ de la fécula de maíz. El análisis de los datos se realizó mediante un diseño completamente al azar, posteriormente se utilizó una comparación de medias de Dúncan. Los datos fueron analizados con el paquete estadístico SAS. (SAS, 1997).

Cuadro 1. Formulaciones empleadas en la elaboración de salchichas con harina de plátano

\section{Harina de plátano (HP)}

\begin{tabular}{ccc}
\cline { 2 - 3 } Experimentos & Proporción & $\mathbf{g ~ 1 0 0 ~ \mathbf { g } ^ { - 1 }}$ carne molida \\
\hline 1 & 0 & 100 \\
2 & 10 & 90 \\
3 & 20 & 80 \\
4 & 30 & 70 \\
5 & 40 & 60 \\
\hline
\end{tabular}

\section{RESULTADOS Y DISCUSIONES}

\section{Análisis de perfil de textura}

Los resultados de este análisis se muestran en el Cuadro 2, donde se observa que la dureza tuvo diferencias significativas $(\mathrm{P}<0.0 .1)$ en las concentraciones de 10, 20 y 40 $\%$ de almidón de plátano, la que no demuestra este comportamiento son las salchichas elaboradas con el 30\%. No fue así en la Cohesividad y la Elasticidad la cual no muestra diferencia significativa $(\mathrm{P}<0.0 .1)$, lo que no sucede en la adhesividad que se muestran cambios al 30 y $40 \%$ la cual aumenta conforme se incrementa el contenido de harina de plátano.

\section{Esfuerzo al corte con navaja Warner-Blazer}

Lo que respecta a la fuerza al corte muestra diferencias significativas en todos los tratamientos con respecto al testigo, tendiente a disminuir conforme se aumenta la incorporación de harina de plátano.

\section{Revista Científica Biológico Agropecuaria Tuxpan 2 (1)}


Sánchez et al., 2014

Cuadro 2. Análisis de Perfil de Textura y Fuerza de Corte para la optimización de las formulaciones.

\begin{tabular}{|c|c|c|c|c|c|c|}
\hline $\begin{array}{c}\text { Carne } \\
\text { molida } \\
(\mathrm{g} 100 \\
\left.\mathrm{g}^{-1}\right)\end{array}$ & $\begin{array}{c}\text { HP } \\
(\mathrm{g} 100 \\
\mathrm{g}-1 \\
\text { harina) }\end{array}$ & $\operatorname{Dureza}(\mathbf{N})$ & Cohesividad & $\begin{array}{l}\text { Adhesividad } \\
\qquad(-\mathrm{cm})\end{array}$ & $\begin{array}{c}\text { Elasticidad } \\
\text { (cm) }\end{array}$ & $\begin{array}{c}\text { Warner } \\
\text { Blatzer } \\
\text { fuerza al } \\
\text { corte }(\mathrm{Kg} / \mathrm{s})\end{array}$ \\
\hline 100.00 & 0.00 & $2.40 \pm 0.78^{a}$ & $0.36 \pm 0.09^{a}$ & $1.01 \pm 0.69^{\mathrm{a}}$ & $0.72 \pm 0.15^{\mathrm{a}}$ & $11.60 \pm 0.49^{a}$ \\
\hline 90.00 & 10.00 & $12.26 \pm 0.80^{\mathrm{ab}}$ & $0.29 \pm 0.09^{\mathrm{a}}$ & $3.32 \pm 0.79^{\mathrm{a}}$ & $0.76 \pm 0.24^{\mathrm{a}}$ & $12.26 \pm 0.87^{\mathrm{a}}$ \\
\hline 80.00 & 20.00 & $8.74 \pm 0.74^{\mathrm{ab}}$ & $0.35 \pm 0.07^{\mathrm{a}}$ & $19.00 \pm 0.56^{\mathrm{a}}$ & $0.68 \pm 0.89^{\mathrm{a}}$ & $8.74 \pm 0.32^{\mathrm{bc}}$ \\
\hline 70.00 & 30.00 & $9.36 \pm 0.69^{\mathrm{a}}$ & $0.37 \pm 0.06^{\mathrm{a}}$ & $63.64 \pm 0.43^{\mathrm{ab}}$ & $0.78 \pm 0.36^{\mathrm{a}}$ & $9.36 \pm 0.73^{b}$ \\
\hline 60.00 & 40.00 & $7.06 \pm 0.96^{\mathrm{b}}$ & $0.34 \pm 0.69^{\mathrm{a}}$ & $88.57 \pm 0.22^{b}$ & $0.70 \pm 0.69^{\mathrm{a}}$ & $7.06 \pm 0.38^{c}$ \\
\hline
\end{tabular}

a, b medias con mismo superíndice en la misma columna no son significativamente diferentes (P $<0,5)$ diferente.

\section{Capacidad de retención de agua}

\section{Análisis de actividad de agua}

Los resultados obtenidos de esta prueba se presentan en la tabla 3, dónde se observa que el único tratamiento que presenta diferencia significativa es el adicionado con $20 \%$ de harina de plátano, y los demás tratamientos presentaron un comportamiento similar con respecto al testigo.
De acuerdo a los datos reportados por esta prueba mostrados en el Cuadro 3, se tiene que esta no tiene diferencia significativa, no causando ningún efecto en esta propiedad, por la utilización de la harina de plátano.

\section{Color}

Los resultados fueron los siguientes: El color fue muy similar al control en el tratamiento 1 con una incorporación de $10 \%$ de harina de plátano este fue disminuyendo con respecto al aumento de incorporación de harina de plátano. La capacidad de retención de agua, fue similar al control en todos los tratamientos. 
Cuadro 3. Aw, capacidad de retención de agua y color en CIE-Lab para la optimización de las formulaciones de salchichas.

\begin{tabular}{|c|c|c|c|c|c|c|}
\hline $\begin{array}{c}\text { Carne } \\
\text { molida } \\
\text { (g } 100 \text { g }^{-1}\end{array}$ & $\begin{array}{c}\text { HP } \\
\text { (g } 100 \\
\mathrm{~g}-1 \\
\text { flour) }\end{array}$ & Aw & $\begin{array}{l}\text { Capacidad } \\
\text { de } \\
\text { retención } \\
\text { de agua } \\
(\%)\end{array}$ & $\begin{array}{c}\text { Luminosidad } \\
\text { L }^{*}\end{array}$ & $\begin{array}{l}\text { Rojo } \\
\mathbf{a}^{*}\end{array}$ & $\begin{array}{c}\text { Amarillo } \\
\qquad \text { b }^{*}\end{array}$ \\
\hline 100.00 & 0.00 & $0.97 \pm 0.89^{\mathrm{a}}$ & $0.973 \pm 0.05^{\mathrm{a}}$ & $64.45 \pm 0.63^{b}$ & $5.41 \pm 0.14^{\mathrm{a}}$ & $9.57 \pm 0.51^{\mathrm{ab}}$ \\
\hline 90.00 & 10.00 & $0.97 \pm 0.40^{\mathrm{ab}}$ & $0.978 \pm 0.77^{\mathrm{a}}$ & $65.50 \pm 0.23^{\mathrm{ab}}$ & $3.35 \pm 0.53^{b}$ & $7.44 \pm 0.16^{b}$ \\
\hline 80.00 & 20.00 & $0.98 \pm 0.57^{a}$ & $.986 \pm 0.63^{\mathrm{a}}$ & $67.04 \pm 0.15^{\mathrm{a}}$ & $3.75 \pm 0.13^{b}$ & $8.60 \pm 1.02^{b}$ \\
\hline 70.0 & 30.00 & $0.96 \pm 0.29^{\mathrm{a}}$ & $0.969 \pm 0.01^{\mathrm{a}}$ & $67.04 \pm 0.09^{\mathrm{a}}$ & $3.13 \pm 0.75^{\mathrm{b}}$ & $11.52 \pm 0.89^{\mathrm{a}}$ \\
\hline 60.0 & 40.00 & $0.96 \pm 0.09^{\mathrm{a}}$ & $0.965 \pm 0.85^{\mathrm{a}}$ & $60.23 \pm 0.89^{c}$ & $5.53 \pm 0.57^{\mathrm{a}}$ & $3.09 \pm 0.75^{\mathrm{c}}$ \\
\hline \multicolumn{6}{|c|}{$\begin{array}{l}\text { medias con mismo superíndice en la misma columna no son significativamente diferentes }(\mathrm{P}<0,5) \\
\text { iferente. }\end{array}$} & $\begin{array}{l}\text { base a esto se tomaron los resultados para la } \\
\text { gráfica siguiente. }\end{array}$ \\
\hline \multicolumn{7}{|c|}{ Análisis Sensorial } \\
\hline $\begin{array}{l}\text { Este ar } \\
3 \text { personas } \\
\text { alchichas, el } \\
\text { ue el número } \\
\text { látano y } 70 \% \\
\text { lar a degusta } \\
\text { ue mejor ca } \\
\text { ales como c }\end{array}$ & $\begin{array}{l}\text { lálisis co } \\
\text { l azar p } \\
\text { tratamien } \\
3 \text { es dec } \\
\text { de fécul } \\
\text { este trat } \\
\text { racterístic }\end{array}$ & $\begin{array}{l}\text { sistió en sele } \\
\text { ra que degus } \\
\text { que se dio a } \\
\text { r, } 30 \% \text { de al } \\
\text { de maíz y s } \\
\text { miento por q } \\
\text { s organolépt }\end{array}$ & $\begin{array}{l}\text { cionar a } \\
\text { aran las } \\
\text { degustar } \\
\text { idón de } \\
\text { deicidio } \\
\text { e fue el } \\
\text { as tuvo }\end{array}$ & \multicolumn{3}{|c|}{$\begin{array}{l}\text { Al } 70 \% \text { de las personas que participaron en este } \\
\text { análisis sensorial les gusto la salchicha y entre } \\
\text { los principales comentarios que se hicieron fue } \\
\text { que el producto es muy bueno, solo que le falta } \\
\text { un poco de color para hacerlo más a atractivo y } \\
\text { que no se distingue el sabor a plátano. Al } 30 \% \\
\text { de esas personas les gusto mucho y decían que } \\
\text { la sensación de fibra en el paladar y sabor es } \\
\text { muy agradable. }\end{array}$} \\
\hline
\end{tabular}

utilizo un formato para análisis sensorial y en 


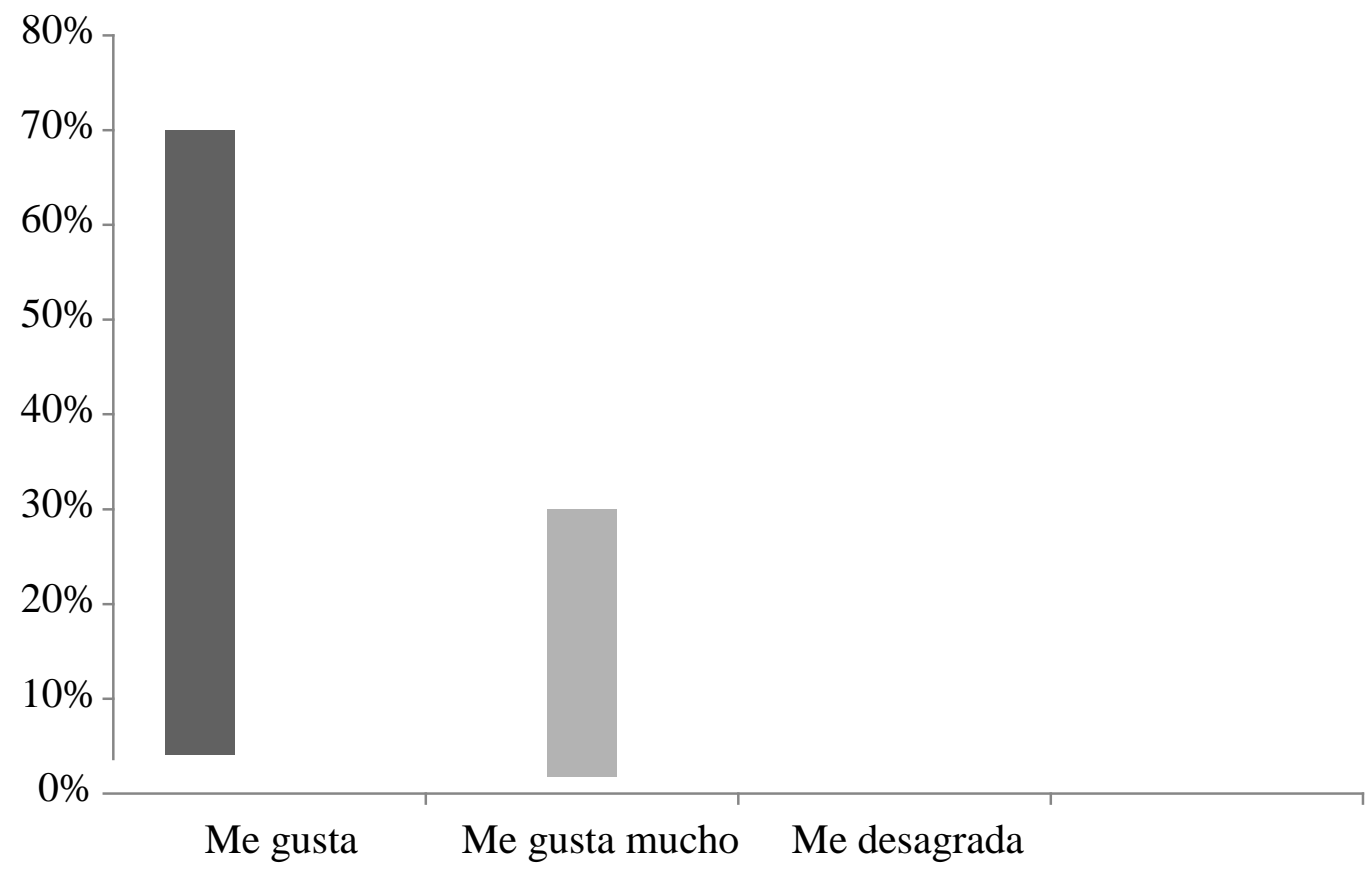

Figura 1. Análisis sensorial de las salchichas adicionadas con harina de plátano macho.

\section{CONCLUSIONES}

Al aumentar el contenido de harina de plátano macho, disminuye la dureza, la fuerza al corte, la Aw, el color de las salchichas. La incorporación de harina de plátano causa una mala incorporación de la grasa al producto debido al rompimiento de la emulsión. El producto fue aceptado por la mayoría de los consumidores por proporcionar un buen sabor.

\section{LITERATURA CITADA}

Anzaldúa, M. A. 1994. La evaluación sensorial de los alimentos en la teoría y en la práctica. Ed. Acribia, Zaragoza, España.
Bello, P. L. 2011. Banana and Mango Flours. Centro de Desarrollo de Productos del IPN, Yautepec, Morelos, México. PP. 237 y 238.

Güemes, V. N. 2007. Utilización de los derivados de cereales y leguminosas en la elaboración de productos cárnicos. Nacameh Vol. 1. No. 2. P 110-117. https://doi.org/10.24275/uam/izt/dcbs/nacameh/2 $007 \mathrm{v} 1 \mathrm{n} 2 /$ guemes

Jauregui, C. A., Regentein, J. M. y Baker, R. C. 1981. A simple centrifugal for measuring expressible moisture, a water- binding for properties of muscle foods. Journal of Food Science 46:12711273. https://doi.org/10.1111/j.1365-2621.1981.tb03038 .x

León, G. J. 2005. Relación de la Microscopia, Calorimetría Diferencial de Barrido y

\section{Revista Científica Biológico Agropecuaria Tuxpan 2 (1)


Textura de Salchichas Bajas en Grasa y Sodio Utilizando Kappa-Carragenia y Otras Sales. Tesis de Licenciatura. Universidad Autónoma del Estado de Hidalgo. Pág. 18.

https://doi.org/10.47212/tendencias_vi_2019_2

Magaldi, A. V. 2007. Tipificación de Chorizos

Producidos en la Región Huasteca del

Estado de Hidalgo. Tesis de Licenciatura. Universidad Autónoma del Estado de Hidalgo. PP. 20.

https://doi.org/10.47212/tendencias_vi_2019_2
Rivera-Ruiz, I. N. 2012. Reducción de grasa y alternativas para su sustitución en productos cárnicos emulsionados, una revisión. NACAMEH Vol. 6, No. 1, pp. $1-14$. https://doi.org/10.24275/uam/izt/dcbs/nacameh/ 2012v6n1/rivera

Copyright (c) 2014 J.F. Sánchez Rodriguez, S. Soto Simental yN. Güemes Vera

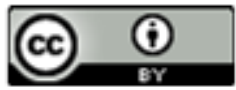

Este texto está protegido por una licencia Creative Commorr 4.0 .

Usted es libre para Compartir —copiar y redistribuir el material en cualquier medio o formato- y Ad aptar el documento —remezclar, transformar y crear a partir del material- para cualquier propósito, inchso para fines comerciales, siempre que cumpla la condición de:

Atribución: Usted debe dar crédito a la obra original de manera adecuada, proporcionar un enlace a la licencia, e indicar si se han realizado cambios. Puede hacerio en cualquier forma razonable, pero no de forma tal que sugiera que tiene el apoyo del licenciante olo recibe por el usoque hace de la obra.

Resumendelicencia - Textocompletodelalicencia 